\title{
Pain Relief Without Medication: Focusing on Pain in Conjunction with Acupuncture Shows More Pain Relief than Acupuncture Alone
}

\author{
Timothy Tong \\ The King's Academy, 562 N Britton Ave, Sunnyvale, CA, 94085, U.S.A.; timothy.tong89010@gmail.com
}

\begin{abstract}
This study investigates whether a focus on pain will increase pain relief during an acupuncture session. For the control group, an acupuncturist performed consecutive acupuncture-only treatments on thirteen patients, and collected their pain rating information before each session. For the experimental group, a patient focused on her pain for three trials and compared those results with her past acupuncture experiences without a focus on pain. In addition, another acupuncturist advised eight to ten of his patients per day over the course of three days to focus only on their pain during treatments and compare their pain relief with their past acupuncture experiences without a focus. Overall, the control group demonstrated that acupuncture reduces pain intensity by $50 \%$ over a period of treatment from acupuncture alone, while the experimental group experienced an additional $10-20 \%$ decrease in pain from concentrating on their pain.
\end{abstract}

KEYWORDS: Biomedical and Health Sciences; Physiological Psychology; Pain Relief; Acupuncture; Focusing on Pain.

\section{Introduction}

In America alone, 50 million adults suffer from chronic pain. ${ }^{1}$ Researchers have developed numerous drugs to rise to the challenge. Alternatively, one can use hot and cold therapy, music therapy, exercise, biofeedback, physical therapy, massage, yoga, and tai chi to reduce pain. ${ }^{2}$ Pain has the ability to draw one's attention involuntarily, which makes it an unavoidable experience among the general population. One might think it is a mistake to pay attention to pain, since it can increase pain intensity. Paying attention to pain only increases pain intensity at first. However, it has been shown that individuals are not be able to concentrate on pain over time. Instead, their mind will float to many things other than pain, which is known as distraction; this leads to pain relief. In contrast, if a person follows instructions to distract themself from the pain at the beginning, eventually their mind may wander back to the pain; this will increase pain intensity. ${ }^{3}$ Although it is hard to pay attention to intense pain, other pain relief methods such as acupuncture may reduce the intensity to make paying attention to pain easier. If acupuncture does not completely remove the pain, one can combine acupuncture along with focusing on pain during acupuncture treatment. There may be additional pain relief from concentrating on the pain.

\section{Literature Review}

Mindfulness meditation is a solution that researchers persist in promoting as an effective attentional activity for pain management. Previous research verifies that mindfulness relieves chronic pain. ${ }^{4}$ Meditation connects to our research because of its association with attention, and this paper focuses on treating pain with an attentional method. Meditation may involve distraction or focus. In traditional meditation programs, one learns to focus on one's breath and then chooses to focus on the area of pain or a different area on their body. ${ }^{5}$

There is brain activity that produces the perception of pain, and that activity increases through paying attention to pain. ${ }^{6}$ However, a study published in 2002 shows that males and clinically anxious chronic pain patients experience less pain when they focus on pain stimuli. ${ }^{7}$ Here, the literature guided us to the debate over whether concentration or distraction is the better way in treating pain. As the reader will see, the effectiveness of one technique over the other depends on the type of pain one experiences. And ultimately, concentration leads to pain relief, only because it keeps one's mind away from pain longer than if one were trying to directly distract oneself from pain. ${ }^{3}$

This principle comes from Wegner's Ironic Process theory. Wegner's theory expanded on the white bear experiment in which it was shown that it is hard to follow the instructions to not think of a white bear. ${ }^{3}$ Essentially, paying attention to something starts an unconscious process that tries to make one pay attention to something else. ${ }^{3}$ Paying attention to pain will start a process that tries to make one pay attention to anything other than the pain. ${ }^{3}$ So, paying attention to something other than pain, known as distraction, will start a process that tries to make one pay attention to anything other than the object of attention-which could include pain. ${ }^{3}$ In Man's Search for Meaning, Viktor Frankl discusses the concept of paradoxical intention that in some ways resembles Wegner's Theory of Ironic Processes. For instance, Frankl discusses how one can get rid of anxieties by trying to worsen them instead of trying to resolve them. ${ }^{8}$ 
Cioffi and Holloway demonstrate Wegner's theory in their experiment where they had participants stick their hands into ice water. ${ }^{9}$ There was a group that paid attention to their hand's pain while it was in the ice water, and a group that distracted themselves from their hand's pain while it was in the ice water.7 Both groups continued to experience pain after taking their hands out of the water, however, the group that focused on the pain experienced pain that faded away faster than the distracted group. ${ }^{9}$

Despite these results, the medical literature suggests limitations to mindfulness in the treatment of pain. For example, meditation affects arthritis pain more than chronic migraines. ${ }^{10}$ This research seeks to improve attentional strategies in the treatment of pain intensity and solve these deficiencies.

A 2012 study on migraine headaches showed that authentic acupuncture reduces more pain than placebo acupuncture that does not follow traditional Chinese medicine practices. ${ }^{11}$ This matters because it is difficult to focus on pain from a migraine due to its high intensity. Acupuncture treatment can make paying attention to such pain easier by lowering the intensity. In agreement, Wegner argues that distraction-not concentration-works better for intense pain. ${ }^{3}$ Alternatively, Wegner suggested that focusing on pain works better for moderate and repeating pain. ${ }^{3}$ So, acupuncture makes concentrating on pain easier and more effective in reducing pain. Here, the idea of synthesizing a pain relief method of focusing on pain along with acupuncture treatment was developed.

A combined treatment with acupuncture and concentration may be best for people who desire pain relief without medication and regularly undergo acupuncture sessions. This experiment will test this new method for greater pain relief. We will then evaluate the results and discuss future research areas.

\section{Interviews: Athletic Trainer}

We interviewed an athletic trainer first. He referred to dry needling when asked of his knowledge of acupuncture. Dry needling involves spinning a needle near an injured part of the body in order to improve blood flow, allowing for a faster recovery from injury. Although this is similar to how acupuncture works, he told us that acupuncture also involves a system in which puncturing one point on the human body triggers a network of other bodily processes. After we brought up the method of paying attention to pain and whether it could increase pain tolerance, he alluded to the transcutaneous electrical stimulation he currently gives to his athletes, recalling that many of his athletes could undergo higher degrees of stimulation as the treatment progressed, because each time their pain tolerance increased. We also asked if paying attention to pain could reduce pain intensity. He responded with another reference to transcutaneous electrical stimulation treatment, explaining that athletes focus on the greater pain coming from the stimulation rather than the lower pain from their sports injury, because the greater pain grabs more attention. Focusing on the pain from the acupuncture needles, he proposed, could increase the distraction from the injury, which would help with pain relief for the injury.

\section{Interviews: Acupuncturist}

We interviewed the acupuncturist second, in order to compare the athletic trainer's point of view on acupuncture with an acupuncture expert's view. We first asked the acupuncturist to explain the acupuncture theory of pain relief. In reply, he referenced nerves around the body that needles could activate to release pain relieving hormonelike substances. We then asked him about the well-known Deqi sensation of acupuncture analgesia. He characterized the sensation as soreness from needling. We brought up other terms in acupuncture, such as meridians and qi. He explained that meridians are channels of energy. The term for energy in acupuncture is qi, and the type of energy acupuncturists refer to is oxygen. There are certain points on meridians that when punctured by needles allow for qi to enter the blood. So, the blood becomes oxygenated, which enhances the healing of wounded cells. When the cells fully heal, pain vanishes. After explaining all of this, he introduced a study that won a Nobel Prize, which explained that greater oxygen levels in body cells improves immunity. This supported the acupuncture theory of pain relief that he had just discussed. We also asked him whether he believes that focusing on pain from injury during acupuncture would increase pain relief. He answered that the acupuncturist should be the one paying attention, so that qi would pass through the acupuncturist into the patient effectively. When asked if acupuncture treatment works in a similar way to transcutaneous electrical stimulation, he responded that they are different, because acupuncture works by Oriental medicine theory and transcutaneous electrical stimulation works by physiological theory.

\section{Interviews: Neurologist}

We asked the neurologist to introduce his profession and his understanding of the pain process. He specializes in treating Parkinson's disease, and believes that pain is one of the main senses, such as taste and touch, that humans experience. Pain follows a pathway from the area of damage to small fibers that lead up to the spinal cord into the thalamus. Both the thalamus and somatosensory cortex are responsible for producing pain perception. We also asked him about pain relief treatment without medication. He mentioned experiments where patients experienced pain relief by focusing on pleasant thoughts. Clinicians also commonly make use of signals from external sources, such as electrical and spinal cord stimulation, to suppress pain signals. In addition, he referred to a few studies done on meditation, where subjects both distracted from and paid attention to their pain.

He also suggested the book, Healing Back Pain, written by John Sarno, that uses a concentration technique to help patients better tolerate their pain. ${ }^{25}$ More specifically, the technique involves uncovering and paying attention to repressed emotion that may be responsible for one's pain.

He also talked about his understanding of acupuncture. Much pain control research has gone into acupuncture. He has observed demonstrations of acupuncture analgesia-that is, pain relief caused by acupuncture-during appendicitis surgery. He agreed with the acupuncturist in that Oriental medicine is incompatible with western medicine. Particularly 
in pain relief treatment, traditional Chinese medicine claims that acupuncture works through a pain network, while western medicine claims that acupuncture works by the release of endorphins, which numbs the pain. He agrees that acupuncturists have already demonstrated the effectiveness of acupuncture convincingly. The neurologist also agreed with the acupuncturist that the effectiveness of acupuncture treatment depends on the practitioner's skill, because different people can produce different results. So, from both points of views, the person providing the treatment themself has a large impact on the result.

As for transcutaneous electrical stimulation, the neurologist referred to spinal cord stimulation. Both methods involve stimulating the nerves leading to the spinal cord close to pain pathways. The external signals disrupt pain signals, which leads to pain relief. While cells mostly interact with each other chemically, cells themselves have electrical qualities, which allows this method to work.

In relation to Wegner's Theory of Ironic Processes, the neurologist commented on how the brain does not like general commands. For example, children break rules when told "not" to do something. This is because the brain is only able to follow specific commands, such as those involving the "doing" of a certain action, but not general commands, such as those involving "not" doing something. Consequently, the brain searches for a specific command to follow, which most of the time turns out to be the forbidden action.

As for paying attention to pain, the neurologist thought that pain intensity would increase. Using signal-to-noise ratio terms from his practice, he stated that neuronal noise-coming from processes in the background of a person's mind-decreases when one focuses on a signal. When the noise decreases, the signal becomes enhanced. So, when pain is the signal, paying attention to pain would increase pain intensity, because the background noise decreases.

Mood can also change pain perception. The neurologist suggested that a calmer mood could lead to a lower pain intensity, because how one thinks about the pain influences how one experiences the pain.

\section{Comparison of ldeas}

Both what the athletic trainer and acupuncturist said primarily helped with a general understanding of how acupuncture treatment works.

The neurologist's views were somewhat more relevant to the paying attention to pain aspect of my experiment. Notably, the signal-to-noise ratio idea does not contradict my experiment. From the beginning, we agreed with Wegner that paying attention to pain should initially increase pain intensity. However, after a period of concentration, the pain intensity will decrease.

The reality that general commands with the word "not" are hard to follow did not cause the pain relief effect observed in my experiment. More specifically, the general command to "not" pay attention to anything other than their pain, would have led some patients to not pay attention to their pain. However, this effect does not apply, because patients followed the direct command to pay attention to their pain.

\section{Results and Discussion}

Figure 1 describes pain ratings versus the number of appointments for the thirteen cases in the control group. The graphs exemplify that, in general, the last session of each treatment period yielded a lower pain intensity than that of the first session, which implies that acupuncture after a period of treatment lowers patient pain intensity, despite pain intensity fluctuations in between. Alternatively, it is possible that the patients were healing naturally too, so further study should be done in this area.

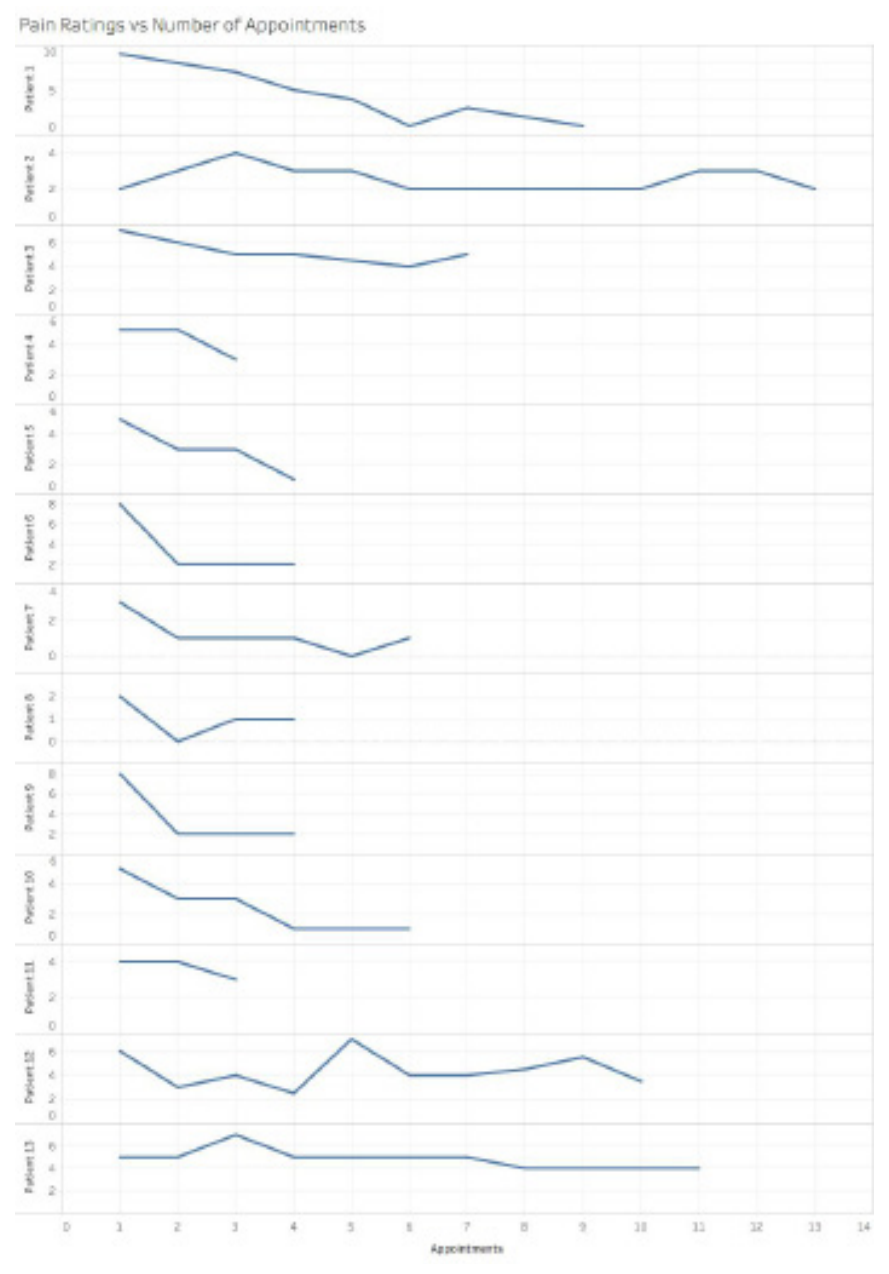

Figure 1. Image produced with Tableau ${ }^{(\mathrm{C}}$

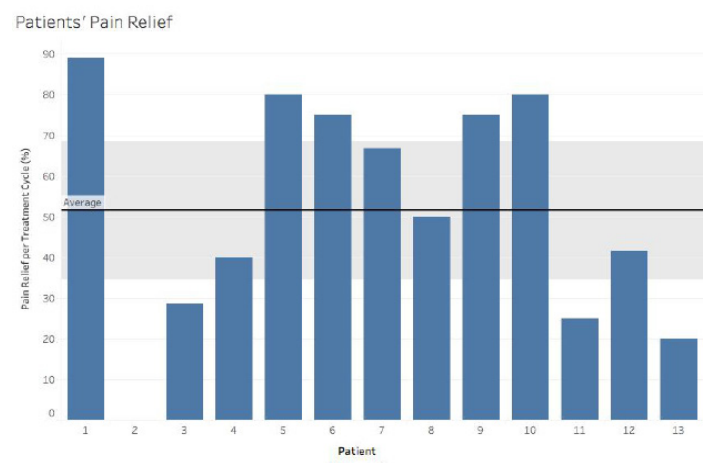

Figure 2. Image produced with Tableau ${ }^{(C)}$ 


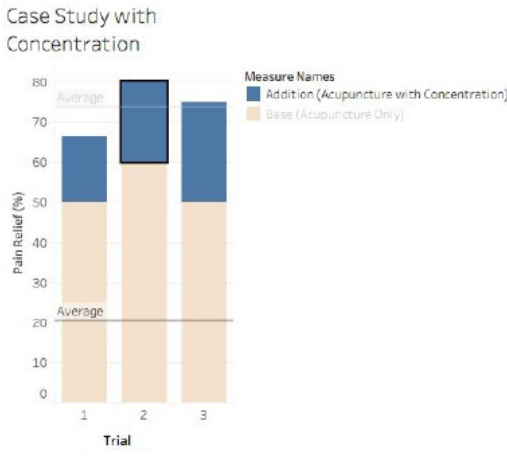

Figure 3. Image produced with Tableau ${ }^{\mathbb{C}}$

As for the first experimental subgroup, Figure 3 is a pain relief comparison of acupuncture treatment with and without concentrating on pain. Referring to Figure 3, the subgroup yielded a total pain relief of $70 \%$ with concentration, and pain relief of $50 \%$ without concentration. So, paying attention to pain contributed $20 \%$ pain relief. The base data was collected from patients' previous experience with acupuncture only.

For the second experimental subgroup, Figure 4 is also a pain relief comparison of acupuncture treatment with and without concentration on pain. The acupuncturist averaged the pain relief results of eight to ten people per day for three days. Referring to Figure 4, the second subgroup yielded a total pain relief of $90 \%$ with concentration, and a pain relief of $80 \%$ without concentration. So, paying attention to pain contributed $10 \%$ pain relief. The acupuncture only data was again gathered from prior experience.

Overall, the experimental group demonstrated a 10-20\% increase in pain relief from paying attention to pain alone.

\section{Results and Discussion}

\section{The Cultural Aspect of Pain}

Pain perception changes between cultures with different values for expressing pain publicly. ${ }^{12}$ For example, stoicism in the West accounts for its subjects' greater pain tolerance when compared to subjects from the East. ${ }^{12}$ Yet, due to the American cultural stereotype that lower economic backgrounds possess a higher pain tolerance, blacks often receive poorer pain relief treatment than whites. ${ }^{13}$ People may try to find meaning in their pain — such as developing views of divine determinism - and depending on their rationalization, they may choose to endure more pain by themselves or they may reach out for help. ${ }^{14}$ The appraisal of pain accounts for some of its intensity. ${ }^{15}$ The biopsychosocial model of pain works by the experience of a nociceptive stimulus, followed by an evaluation of the threat, somatic responses, and then an adaptation of behavior. ${ }^{16}$ Culture influences the evaluation process-whether a person perceives their situation as dangerous or normalwhich affects the intensity of the pain experience. ${ }^{17}$

Perhaps with more Chinese people participating in traditional acupuncture, the results demonstrated a greater pain relief from the treatment, as they evaluated the pain experience with an expectation that their pain would decrease from it. This study did not investigate differences in pain relief from the treatment between ethnic groups. Maybe one could consider comparing a different ethnic group with a sample of Chinese people, since it appears that Chinese people tend to believe in the effectiveness of this form of treatment and participate in it more than any other people.

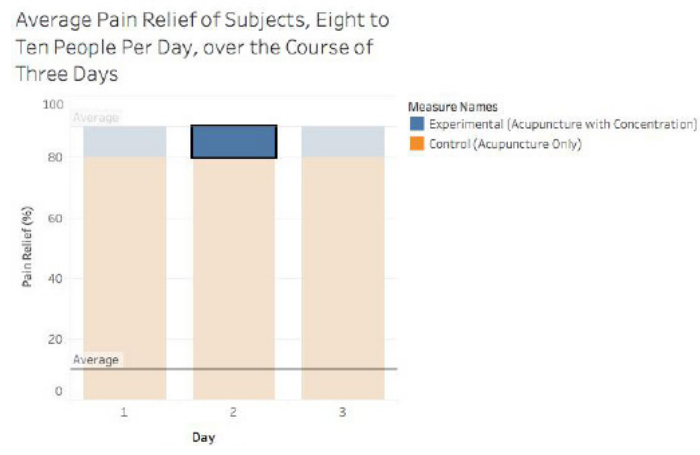

Figure 4. Image produced with Tableau ${ }^{(C)}$

\section{The Psychological Aspect of Pain}

While culture influences whether or not a person perceives pain as dangerous, psychology explains how these emotional responses affect the pain perception. Specifically, one's fear of pain could increase perceived pain intensity. ${ }^{18}$ Researchers in this area refer to the activity of fearing pain as "catastrophization". ${ }^{19}$ The neurologist I interviewed supported this phenomenon, in addition, mentioning that a peaceful mood could lower perceived pain intensity. Similarly, acceptance therapy aims to change the psychological reaction of patients to their chronic pain, in order to help them cope with pain as much as possible. ${ }^{18}$

The patients lied down on a cushion in a silent room, with a warm beam of light on their back. Maybe these surroundings elicited a peaceful mood from most of the patients, which perhaps aided the reduction of pain intensity during acupuncture treatment. An investigation into the pain relief from emotional responses could involve two groups of patients undergoing acupuncture, one group with a beam of light and the other without.

\section{The Biological Aspect of Pain}

The actual biological process behind the pain sensation is more significant than the psychosocial evaluation process. The neurologist we interviewed explained the biological process, referring to the journey of a pain signal from an injury through the nerves to the spinal cord, then mediated with small fibers leading up to the thalamus. After entering the somatosensory cortex, the signal activates the nociceptors in the brain responsible for producing the perception of pain. ${ }^{20}$ However, despite this common interpretation, other parts of the brain are also responsible for the perception of pain. ${ }^{21}$ For example, research suggests that acupuncture analgesia works by changing pain related activity in the amygdala. ${ }^{22}$

The athletic trainer we interviewed introduced to us the gate control theory of pain. Essentially, a gate in the spinal cord 
prevents innocuous stimuli from entering the thalamus, and allows in noxious stimuli, the latter producing pain perception. ${ }^{23}$ The gate is able to differentiate between innocuous and noxious stimuli because innocuous stimuli travel through large fibers, while noxious stimuli travel through small fibers. ${ }^{23}$ See Figures 5 and 6 below:

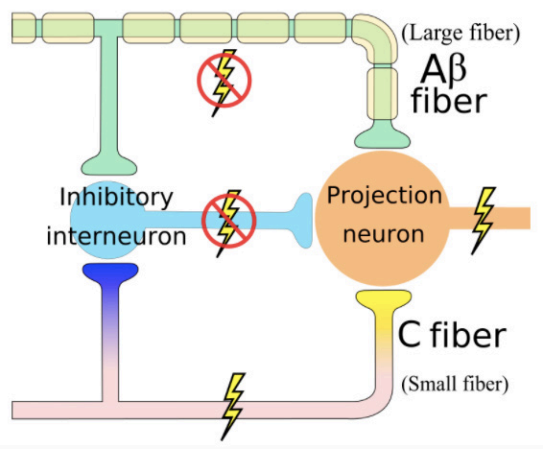

Figure 5. Figure altered from commons.wikimedia.org.

Figure 5 shows the inhibitory interneuron and projection neuron at the spinal cord. The inhibitory interneuron is inactive when there are no innocuous stimuli coming through the large fiber $A \beta$. Otherwise, when there are innocuous stimuli coming in, the inhibitory interneuron is active, and closes the gate at the projection neuron, so that stimuli do not reach the thalamus. Alternatively, when the inhibitory interneuron is off, the projection neuron is active. So, stimuli enter the thalamus, and pain sensation occurs.

Figure 5 depicts a situation where there are incoming noxious stimuli but no innocuous stimuli. Because there are no innocuous stimuli entering through the large fiber, the inhibitory interneuron is off. The crossed-out lightning bolts signify their inactivity. Because the inhibitory interneuron is off, the projection neuron is on. The lightning bolt next to the projection neuron signifies activity. The small fiber $\mathrm{C}$ has noxious stimuli traveling through its pathway. The lightning bolt next to the small fiber signifies activity. Since the gate at the projection neuron is open, noxious stimuli is arriving at the thalamus, so that pain sensation occurs.

Figure 6 depicts a situation where there are both noxious and innocuous stimuli going into the system. Innocuous stimuli are traveling through the large fiber $A \beta$, as symbolized by the lightning bolt. Because innocuous stimuli are coming in, the inhibitory interneuron is active, also symbolized by a lightning

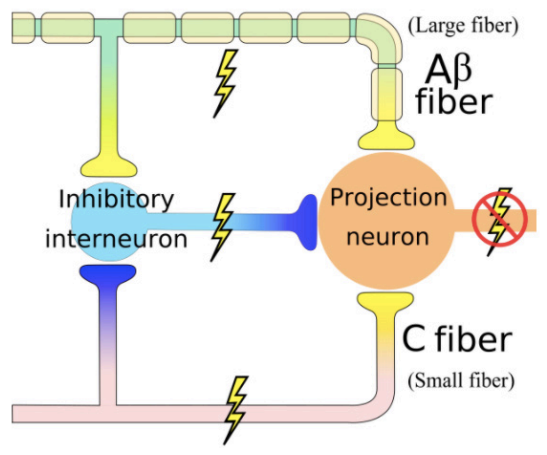

Figure 6. Figure altered from commons.wikimedia.org. bolt. Since the inhibitory interneuron's function is to close the gate at the projection neuron, the projection neuron is inactive in this case, symbolized by a crossed-out lightning bolt. With the gate closed, even though the small fiber $\mathrm{C}$ has noxious stimuli traveling through its pathway, none of the stimuli enter the thalamus, and pain sensation does not occur.

Western clinicians commonly explain acupuncture analgesia based on gate control theory. The acupuncture needles send innocuous stimuli through large fibers to close the gate at the spinal cord, which in effect also blocks noxious stimuli from entering the thalamus. ${ }^{24}$ Other research suggests that electrical transmission from acupuncture needles releases the pain relieving neurochemicals that are responsible for acupuncture analgesia. ${ }^{24}$ This is similar to how pain relief from electrical needling works. ${ }^{24}$

\section{- Conclusion}

Acupuncture treatment is an effective alternative to pain relief drugs. One can also expect an additional 10-20\% pain relief from paying attention to pain during an acupuncture session when combined with mind exercises that focus on the pain.

Methods

The difference between the control and experimental groups was that, during acupuncture treatment, the control group did not focus on their pain, while the experimental groups did focus on their pain.

We started our investigation with a control group in an acupuncture clinic. The acupuncturist provided the information. We randomly sampled thirteen patients. Patients reported pain ratings in certain areas on their body using a form provided by the acupuncturist. The pain rating scale on the form was from an intensity of one to ten. Pain ratings were recorded before each session of a treatment period.

Treatment periods lasted until the patient decided they no longer needed the acupuncture. Pain ratings right after each session were not available. Many patients reported multiple types of pain at different times during their treatment period. One type of pain always took a longer part of the treatment period, so only this part of the treatment period was considered.

For the experimental group, there were two subgroups. The first experimental subgroup was a study of one patient for three trials in the control group clinic. For the second experimental subgroup, another acupuncturist treated eight to ten people per day over the course of three days. In both clinics, patients focused on their pain from their injury during the acupuncture treatment. Each patient then reported their pain relief while focusing on pain. They also compared their pain relief from acupuncture alone using a previous treatment experience.

There were two experimental groups because my first experiment was only with one willing participant. A survey was used for the second experiment to enlarge the sample size. 


\section{Experimental Limitations}

The experiment measured correlational rather than causational results due to possible confounding variables. One limitation in my experimental group is that patients used their memory to report pain relief from past acupuncture alone experiences. A person's memory is not always reliable. A more accurate experiment can record the pain relief from acupuncture alone immediately after an acupuncture session without paying attention to pain. Later, another session can record the pain relief experienced by patients from a combined treatment of acupuncture with paying attention to pain. As for the control group, it would have been improved if we had access to patient pain ratings immediately after each session

\section{Future Direction}

It is possible that acupuncture treatment reduces more pain intensity for some types of pain than others. One could test if this is the case by comparing the pain relief that patients of different types of pain experience from acupuncture treatment. The experiment will have to consider the area of the pain on the body, as well as the pain's cause, duration, and symptoms.

It is also worthwhile to explore whether paying attention to pain, or distracting from pain, relieves pain intensity. This may relate to the patient's original pain intensity. The experiment needs one group of patients suffering high intensity pain, and another group of patients suffering medium to low intensity pain. Within each group, there are two subgroups to explore paying attention to pain or distracting from pain. The experimenters will then compare the pain relief experienced from the methods with respect to the initial level of pain intensity.

It would be useful to know how long it takes for a person to drift into unrelated thoughts, after instructed to pay attention to something. In the same experiment, it would be interesting to find out how many times participants drift back and forth between distraction and paying attention, and the percentage of time spent in each area.

The neurologist raised that researchers have used fMRIs to objectively quantify reported pain ratings with brain activity. Another experiment could use this technology to understand the neurological processes behind the pain relief from paying attention to pain.

\section{- Acknowledgements}

Thanks to Mrs. Alice Wu for guiding my research and reaching out to people who could help. Thanks to Mrs. Alex Park for mentoring me over the course of my research and offering her expertise in psychology. Thanks to Mr. Darren Ngo, Dr. Jun Cao, and Dr. Nengchun Huang for spending time with me in interviews and contributing their thoughts on pain relief.

\section{References}

1. "Anson, Pat. CDC: 50 Million Americans Have Chronic Pain. Pain News Network, September 13, 2018. https://www.painnewsnetwork.org/ stories/2018/9/13/cdc-50-million-americans-have-chronic-pain (accessed August 4, 2020).

2. Harvard Health Publishing. Pain Relief Without Drugs or Surgery. https://www.health.harvard.edu/pain/pain-relief-without-drugs-or- surgery (accessed August 4, 2020).

3. Wegner, Daniel M.; Schneider, David J.; Carter III, Samuel R.; White, Teri L. Paradoxical Effects of Thought Suppression. J. Pers. Soc. Psychol. [Online] 1987, 53 (1), 5-13. Demenzemedicinagenerale. http://www.demenzemedicinagenerale.net/images/pdf/

Tought $\% 20$ suppression $\% 20$ (orso\%20bianco).pdf (accessed August 4, 2020).

4. Morone, Natalia E.; Lynch, Cheryl S.; Greco, Carol M.; Tindle, Hilary A.; Weiner, Debra K. "I Felt like a New Person."

The Effects of Mindfulness Meditation on Older Adults with Chronic Pain: Qualitative Narrative Analysis of Diary Entries. J. Pain [Online] 2008, 9 (9), 841-848. U.S. National Library of Medicine. https://www.ncbi.nlm.nih.gov/pubmed/18550444 (accessed August 4, 2020).

5. Kabat-Zinn, John. An Outpatient Program in Behavioral Medicine for Chronic Pain Patients Based on the Practice of Mindfulness Meditation: Theoretical Considerations and Preliminary Results. Gen. Hosp. Psychiatry [Online] 1982, 4 (1), 33-47. Sophro-Axe. http://www.sophro-axe.fr/fs/Articles_scientifiques_Mindfulness/ 6lwfgMindfulness_chronic_pains_kabatzinn_mbsr_1982.pdf (accessed August 4, 2020)

6. Bantick, Sussana J.; Wise, Richard G.; Ploghaus, Alexander; Clare, Stuart; Smith, Stephen M.; Tracey, Irene. Imaging How Attention Modulates Pain in Humans Using Functional MRI. Brain [Online] 2002, 125 (2), 310-319. Academic OUP. https://academic.oup.com/ brain/article/125/2/310/296978 (accessed August 4, 2020).

7. Villemure, Chantal; Bushnell, Catherine M. Cognitive Modulation of Pain: How Do Attention and Emotion Influence Pain Processing? Pain [Online] 2002, 95 (3), 195-199. https://doi.org/10.1016/ S0304-3959(02)00007-6 (accessed August 4, 2020).

8. Frankl, V.E. Man's Search for Meaning: An Introduction to Logotherapy; Beacon Press: Boston, 2006

9. Cioff, Deliax; Holloway, James. Delayed Costs of Suppressed Pain. J. Pers. Soc. Psychol [Online] 1993, 64 (2), 274-282. American Psychological Association. https://psycnet.apa.org/doiLanding?doi=10.1037\%2F0022-3514.64.2.274 (accessed August 4, 2020).

10.Rosenzweig, Steven; Greeson, Jeffrey M.; Reibel, Diane K.; Green, Joshua S.; Jasser, Samar A.; Beasley, Denise. Mindfulness-Based Stress Reduction for Chronic Pain Conditions: Variation in Treatment Outcomes and Role of Home Meditation Practice. J. Psychosom. Res. [Online] 2010, 6 (1), 29-36. ScienceDirect. https://doi.org/10.1016/j.jpsychores.2009.03.010 (accessed August 4, 2020).

11. National Center for Complementary and Integrative Health. Acupuncture: In Depth. https://nccih.nih.gov/health/acupuncture /introduction (accessed August 4, 2020).

12. Anderson, Steven R.; and Reynolds Losin, Elizabeth A. A Sociocultural Neuroscience Approach to Pain. Cult. Brain [Online] 2016, 5 (1), 14-35. SpringerLink. https://doi.org/10.1007/s40167-016-0037-4 (accessed August 4, 2020).

13.Peacock, Sue; Patel, Shilpa. Cultural Influences on Pain. Br. J. Pain [Online] 2008, 1 (2), 6-9. Sage Journals. https://doi.org/10.1177/204946370800100203 (accessed August 4, 2020).

14.Lovering, Sandy. Cultural Attitudes and Beliefs About Pain. J. Transcult. Nurs. [Online] 2006, 17 (4), 389-95. Sage Journals. https://doi.org/10.1177/1043659606291546 (accessed August 4, 2020).

15.Moseley, G. Lorimer. Reconceptualising Pain According to Modern Pain Science. Phys. Ther. Rev. [Online] 2007, 12 (3), 169-78. Taylor \& Francis Online. https://doi.org/10.1179/108331907x223010 (accessed August 4, 2020).

16. Frankl, V.E. Man's Search for Meaning: An Introduction to Logotherapy; Beacon Press: Boston, 2006 Garland, Eric L. Pain Processing in the Human Nervous System: A Selective Review of Nociceptive and Biobehavioral Pathways. Prim. Care [Online] 2012, 39 (3), 561-71. ScienceDirect. https://dx.doi.org/10.1016\%2Fj.pop.2012.06.013 (accessed August 4, 2020).

17. Bendelow, Gillian A.; Williams, Simon J. Transcending the Dualisms: towards a Sociology of Pain. Sociol. Health. Illn. [Online] 199517 (2), 139-65. Wiley Online Library. https://onlinelibrary.wiley.com/ doi/epdf/10.1111/j.1467-9566.1995.tb00479.x (accessed August 4, 2020). 
18.Morley, S. Psychology of Pain. Br. J. Anaesth. [Online] 2008, 101 (1), 25-31. https://doi.org/10.1093/bja/aen123 (accessed August 4, 2020).

19.Campbell, Claudia M.; Edwards, Robert R. Mind-Body Interactions in Pain: the Neurophysiology of Anxious and Catastrophic Pain-Related Thoughts. Transl. Res [Online] 2009, 153 (3), 97-101. https://doi.org/10.1016/j.trsl.2008.12.002 (accessed August 4, 2020).

20. Stucky, Cheryl L; Gold, Michael S.; Zhang, Xu. Mechanisms of Pain. Proc. Natl. Acad. Sci. U.S.A. [Online] 2001, 98 (21), 11845-11846. https://doi.org/10.1073/pnas.211373398 (accessed August 4, 2020).

21. Coghill, Robert C.; Sang, Christine N.; Maisog, Jose Ma.; Iadarola, Michael J. Pain Intensity Processing Within the Human Brain: A Bilateral, Distributed Mechanism. J. Neurophysiol. [Online] 1999, 82 (4), 1934-1943. https://doi.org/10.1152/jn.1999.82.4.1934 (accessed August 4, 2020).

22. Qin, Wei; Tian, Jie; Bai, Lijun; Pan, Xiaohong; Yang, Lin; Chen, Peng; Dai, Jianping; Ai, Lin; Zhao, Baixiao; Gong, Qiyong; Wei, Wang; von Deneen, Karen M.; Liu Yijun. FMRI Connectivity Analysis of Acupuncture Effects on an Amygdala-Associated Brain Network. Mol. Pain [Online] 2008, 4 (January 1, 2008). Sage Journals. https://doi. org/10.1186/1744-8069-4-55 (accessed August 4, 2020).

23.Mendell, Lorne M. Constructing and Deconstructing the Gate Theory of Pain. U.S. National Library of Medicine, February. Pain [Online] 2014, 155 (2), 210-216. https://www.ncbi.nlm.nih.gov/pmc/ articles/PMC4009371/ (accessed August 4, 2020).

24.PUlett, George A.; Han, Jisheng; Han, Songping. Traditional and Evidence-Based Acupuncture: History, Mechanisms, and Present Status. South. Med. J. [Online] 1998, 91 (12), 1115-11120. Geocities. http://www.geocities.ws/rudhyad/Download/Evidence_Based_ Acupuncture.pdf (accessed August 4, 2020).

25.Sarno, J.E. Healing Back Pain: The Mind-Body Connection; Warner Books: New York, 1991.

\section{Authors}

Timothy Tong is a twelfth grader attending The King's Academy. He is interested in Psychology and Cognitive Neuroscience. 